\title{
TITLE:
}

\section{RATIONAL COHOMOLOGY OF WITT GROUPS}

$\operatorname{AUTHOR}(\mathrm{S})$ :

Peterson, Chariya; Yagita, Nobuaki

\section{CITATION:}

Peterson, Chariya ...[et al]. RATIONAL COHOMOLOGY OF WITT GROUPS. 数理解析研究所講究録 1993, 838: 110-122

ISSUE DATE:

1993-05

URL:

http://hdl.handle.net/2433/83504

RIGHT: 


\title{
RATIONAL COHOMOLOGY OF WITT GROUPS
}

\author{
Chariya Peterson and Nobuaki Yagita \\ February 3, 1993
}

Let $k$ be an algebraically closed field of characteristic $p$ and for each $n>0$ let $W(n)$ denote the group of Witt vectors of length $n$. $W(n)$ is a commutative algebraic group. For reference, see Jacobson [2], Serre [6]. One of the important properties of the Witt groups is the following: Every commutative algebraic $k$-group whose underlying variety is an affine space is a homomorphic image of products of $W(n)$. We compute the rational cohomology of $W(n)$ for $n \geq 2$.

$$
\mathrm{H}^{*}(W(n), k)=S\left(\left(V^{n-1 *}\right)^{-1} \beta L^{\#}\right) \otimes E\left(R^{n-1 *} L^{\#}\right),
$$

where $\beta$ is the Bockstein, $V$, the shift and $R$ the restriction homomorphism and where $L^{\#}$ is the graded dual of the restricted Lie algebra $\operatorname{End}\left(G_{a}\right)$ identified with the first cohomology group $\mathrm{H}^{1}\left(G_{a} ; k\right) \cong \oplus k x^{\left[p^{i}\right]}$. We also show the existence of the higher Bockstein for 1-dimensional cohomology classes of algebraic groups. As an application, we compute the rational cohomology of a family of commutative unipotent groups $V(n)$ and discuss the connection of these cohomology rings with that of the Steenrod algebra.

\section{The ring of Witt vectors}

Let $W=\mathbb{Q}\left(x_{i}, y_{j}, z_{k}\right), 0 \leq i, j, k<m$ be a polynomial $\mathbb{Q}$ algebra and let $W_{n}=W \times \cdots \times W$ be an $n$-fold product of $W$ with componentwise addition " + " and multiplication ".". Define a new addition " $\oplus$ " and multiplication " $\odot$ " on $W_{n}$ as follows:

$$
\begin{aligned}
& a \oplus b=\phi^{-1}(\phi a+\phi b) \\
& a \odot b=\phi^{-1}(\phi a \cdot \phi b),
\end{aligned}
$$

where, for $a=\left(a_{0}, \ldots, a_{m-1}\right), \phi a=\left(\phi a_{0}, \ldots, \phi a_{m-1}\right)$ with $\phi a_{r}=\sum_{0}^{r} p^{i} a_{i}^{p^{r-i}}$. It's inverse $\phi^{-1}$ is defined inductively as: $\phi^{-1} a_{0}=a_{0}$ and $\phi^{-1} a_{r}=\frac{1}{p^{r}}\left(a_{r}-\sum_{i=0}^{r-1} p^{i}\left(\phi^{-1} a_{i}\right)_{i}^{p^{r-i}}\right)$. The triple $\left(W_{n}, \oplus, \odot\right)$ is a commutative ring over $\mathbb{Q}$ with $1=(1,0, \ldots, 0)$ as identity and $(0, \ldots, 0)$ as zero element. The map $\phi: W_{n} \rightarrow W_{n}$ is a ring isomorphism from $\left(W_{n}, \oplus, \odot\right)$ onto $\left(W_{n},+, \cdot\right)$.

Consider generic vectors $x=\left(x_{0}, \ldots, x_{m-1}\right)$ and $y=\left(y_{0}, \ldots, y_{m-1}\right)$, with $x_{i}$ and $y_{i}$ indeterminate as above, then each component of $x \oplus y$ and $x \odot y$ are in fact a polynomial with 
integral coefficient. $(x \oplus y)_{r},(x \odot y)_{r} \in \mathbb{Z}\left[x_{0}, y_{0}, \ldots, y_{r}\right]$ for $0 \leq r<m$. For example:

$$
\begin{aligned}
& (x \oplus y)_{0}=x_{0}+y_{0} \\
& (x \oplus y)_{1}=x_{1}+y_{1}-\frac{1}{p} \sum_{1}^{p-1}\left(\begin{array}{l}
p \\
i
\end{array}\right) x_{0}^{i} y_{0}^{p-i} \\
& (x \odot y)_{0}=x_{0} y_{0} \\
& (x \odot y)_{1}=x_{0}^{p} y_{1}+x_{1} y_{0}^{p}+p x_{1} y_{1} .
\end{aligned}
$$

For an arbitrary commutative ring $A$ of characteristic $p$, let $W_{m}(A)$ be the set of mtuples $\left(a_{0}, \ldots, a_{m-1}\right)$ with $a_{i} \in A$ and with addition and multiplication defined via the polynomials $(x \oplus y)_{r}$ and $(x \odot y)_{r}$ as follows: For any three elements $a, b, c \in W_{m}(A)$, let $s: \mathbb{Z}\left[x_{i}, y_{i}, z_{i}\right] \rightarrow W_{m}(A)$ be the map sending $x_{i}, y_{i}, z_{i}$ to $a_{i}, b_{i}, c_{i}$ respectively. Then $W_{m}(A)$ becomes an associative commutative ring of characteristic $p$ with $(a \oplus b)_{r}=$ $s(x \oplus y)_{r}$ and $\left.(a \odot b)_{r}=s(x \odot y)_{r}\right)$, called the ring of Witt vectors of length $m$. In fact, $W_{m}$ is a functor from commutative rings of characteristic $p$ to commutative rings. The prime ring of $W_{m}(A)$ is isomorphic to $\mathbb{F}_{p^{m}}$. It consists of Witt vectors with coefficients in $\mathbb{F}_{p}$, the prime ring of $A$.

\section{Witt groups}

The underlying abelian group of $W_{n}$, denoted by $W(n)$ is a commutative algebraic group. It is commonly known as the Witt group of dimension, or length, $n$. There are natural homomorphisms among $W(n)$ for various $n \geq 1$ :

(1) The Frobenius homomorphism:

(2) The restriction homomorphism:

(3) The shift homomorphism

$$
\begin{array}{ll}
F: W_{m} \rightarrow W_{m}: & F(a)=\left(a_{0}^{p}, \ldots, a_{n-1}^{p}\right) \\
R: W_{m} \rightarrow W_{m-1}: & R(a)=\left(a_{0}, \ldots, a_{n-2}\right) \\
V: W_{m} \rightarrow W_{m+1}: & V(a)=\left(0, a_{0}, \ldots, a_{n-1}\right)
\end{array}
$$

$R, F$ and $V$ commute with each other and their product $R F V$ is multiplication by $p$.

Similar to the ring $W_{n}$, the Hopf algebra associated to $W(n)$ is constructed first in characteristic 0 , then followed by reduction $\bmod p$. Over the field of rational numbers $\mathbb{Q}$, consider the associated algebra $\mathbb{Q}\left[y_{0}, \cdots, y_{n-1}\right]$ of the additive $\mathbb{Q}$-vector group $G_{a}^{n}$. For $0 \leq j<n$ let $x_{j}=\psi\left(y_{i}\right)=p^{j} y_{j}+p^{j-1} y_{j-1}^{p}+\cdots+y_{0}^{p^{j-1}}$. The $\mathbb{Z}$ lattice $\mathbb{Z}\left[x_{0}, \ldots, x_{n-1}\right]$ of $\mathbb{Q}[V]$ generated by the $x_{i}$ 's is closed under comultiplication, counit and antipode. That is $\psi$ is an automorphism on $\mathbb{Q}\left[y_{0}, \ldots, y_{n-1}\right]$ whose restriction to the $\mathbb{Z}$-lattice $\mathbb{Z}\left[y_{0}, \ldots, y_{n-1}\right]$ induces a Hopf algebra structure on its image $\mathbb{Z}\left[x_{0}, \ldots, x_{n-1}\right]$. For any field $k$ of characteristic $p>0, W(n)$ is defined to be the algebraic group associated to $\mathbb{Z}\left[x_{0}, \ldots, x_{n-1}\right] \otimes k=k\left[x_{0}, \ldots, x_{n-1}\right]$. The generator $x_{i}$ is the function $x_{i}(a)=a_{i}$ for $a \in W(n)(A)$. The first few examples are 
$\triangle x_{0}=x_{0} \otimes 1+1 \otimes x_{0}$ and from (2)

$$
\triangle x_{1}=x_{1} \otimes 1+1 \otimes x_{1}-\frac{1}{p} \sum\left(\begin{array}{c}
p \\
i
\end{array}\right) x_{0}^{i} \otimes x_{0}^{p-i}
$$

\section{Cohomology of $W(n)$}

Let $G$ be an algebraic group defined over a field $k$ and $k[G]$ be its coordinate algebra. For a $G$ module $M$, the rational cohomology $\mathrm{H}^{*}(G ; M)$ is the homology of the cobar complex.

$$
C^{n}(G, M)=M \otimes I^{n} ; \quad I \text { is the augmentation ideal of } k[G],
$$

with the coboundary $\partial^{i}: C^{i}(G, M) \rightarrow C^{i+1}(G, M)$

$$
\partial^{i}\left(f_{0} \otimes \ldots \otimes f_{i}\right)=\sum_{j=0}^{i}(-1)^{j} f_{0} \otimes \ldots \otimes\left(\Delta\left(f_{j}\right)-f_{j} \otimes 1-1 \otimes f_{j}\right) \otimes \ldots \otimes f_{i}+f_{0} \otimes \ldots \otimes f_{i} \otimes 1
$$

Let $k\left[G_{a}\right]=k[x]$ be the associted algebra of the additive algebraic group $G_{a}$. The rational cohomology of $G_{a}$ is given (see Cline, Parshall, Scott and van der Kallen, 4.1 in [1]),

$$
\mathrm{H}^{*}\left(G_{a} ; k\right) \cong \begin{cases}S\left(\beta L^{\#}\right) \otimes E\left(L^{\#}\right) & \text { for } p \geq 3 \\ S\left(L^{\#}\right) & \text { for } p=2\end{cases}
$$

where $L$ is the restricted Lie algebra $\operatorname{End}\left(G_{a}\right)$, which can be identified with the infinite sum $\oplus_{i=0}^{\infty} k x^{p^{i}}$. Let $x(i)$ denotes the dual basis to $x^{p^{i}}$ and identify it with the first cohomology class of $1 \otimes x^{p^{i}} \in C^{1}\left(G_{a}, k\right) . S(-)$ and $E(-)$ are the symmetric and exterior algebra and $\beta$ denotes the (algebraic) Bockstein induced from the map $\bar{\beta}: C^{1}\left(G_{a}, k\right) \rightarrow C^{2}\left(G_{a}, k\right)$. For any monomial $x^{i}$

$$
\bar{\beta} x^{i}=-\frac{1}{p} \sum_{j=1}^{p-1}\left(\begin{array}{l}
p \\
j
\end{array}\right) x^{i j} \otimes x^{i(p-j)}
$$

Remark 3.1 For $p=2$ we have $\beta x(i)=x(i)^{2}$. However for $p \geq 3 \beta$ is not the usual Bockstein $\tilde{\beta}$ in the ordinary cohomology, which is induced from the long exact sequence from the extension

$$
0 \rightarrow k \rightarrow W(2)(k) \rightarrow k \rightarrow 0,
$$

but it is $\tilde{\beta} P^{0}$ (for detail see the appendix A1.5.2 in Ravenel [5]). Indeed, for $\mathrm{H}^{*}\left(G_{a} ; k\right)$, $P^{0}$ is the Frobenius homomorphism in $L, P^{0} x(i)=x(i+1)$ and $\beta x(i)=\tilde{\beta} x(i+1)$. 
In terms of $x(i)$ and $\beta x(i):=y(i+1)$ we write

$$
\mathrm{H}^{*}\left(G_{a} ; k\right) \cong \begin{cases}\bigotimes_{i=0}^{\infty} k[y(i+1)] \otimes E(x(i)) & \text { for } p \geq 3 \\ \bigotimes_{i=0}^{\infty} k[x(i)] & \text { for } p=2\end{cases}
$$

Now we consider the cohomology of $W(n)$. For each pair of positive integers $n, m$, the homomorphisms $R$ and $V$ induce an extension

$$
0 \rightarrow W(m) \rightarrow W(n+m) \rightarrow W(n) \rightarrow 0 .
$$

In particular, for $n-1$ and 1 we have the extension

$$
0 \rightarrow G_{a} \rightarrow W(n) \rightarrow W(n-1) \rightarrow 0
$$

which corresponds to the coextension of Hopf algebras:

$$
k\left[x_{n-1}\right] \leftarrow k\left[x_{0}, \ldots, x_{n-1}\right] \leftarrow k\left[x_{0}, \ldots, x_{n-2}\right],
$$

To compute $\mathrm{H}^{*}(W(n) ; k)$ for $n \geq 2$ we apply the Hochschild-Serre's spectral sequence

$$
E_{2}^{*, *}(n)=\mathrm{H}^{*}\left(W(n-1) ; \mathrm{H}^{*}\left(G_{a} ; k\right)\right) \Longrightarrow \mathrm{H}^{*}(W(n) ; k) .
$$

For $n=2$ and $p \geq 3$

$$
E_{2}^{*, *}(2) \cong \bigotimes_{i=0}^{\infty} k\left[y_{0}(i+1), y_{1}(i+1)\right] \otimes E\left(x_{0}(i), x_{1}(i)\right)
$$

The differential in $C^{*}(W(n), k)$ is given by (3), (4) and (5)

$$
\partial_{1} x_{1}^{p^{i}}=\Delta x_{1}^{p^{i}}-\left(x_{1}^{p^{i}} \otimes 1-1 \otimes x_{1}^{p^{i}}\right)=\beta x_{0}^{p^{i}}
$$

So the induced differential in the spectral sequence is $d_{2} x_{1}(i)=y_{0}(i+1)$. Hence

$$
E_{3}^{*, *}(2) \cong \bigotimes_{i=0}^{\infty} k\left[y_{1}(i+1)\right] \otimes E\left(x_{0}(i)\right)
$$

By Cartan-Serre's transgression theorem (see the appendix A.1.5.2 in [5])

$$
d_{3} y_{1}(i+1)=d_{3}\left(\tilde{\beta} P^{0} x_{1}(i)\right)=\tilde{\beta} P^{0} d_{2} x_{1}(i)=\tilde{\beta} P^{0} y_{0}(i+1)=\tilde{\beta} y_{0}(i+2)=0 .
$$

Therefore $E_{3}^{*, *}(2) \cong E_{\infty}^{*, *}(2)$ and we have just proved the following theorem for $n=2$.

Theorem 3.2 (Compare VII, 9, Lemma \& in [6]). For any integer $n \geq 1$,

$$
\begin{aligned}
\mathrm{H}^{*}(W(n) ; k) & \cong \bigotimes_{i=0}^{\infty} k\left[y_{n-1}(i+1)\right] \otimes E\left(x_{0}(i)\right) & & \text { for } p \geq 3 \\
& \cong \bigotimes_{i=0}^{\infty} k\left[x_{n-1}^{2}(i)\right] \otimes E\left(x_{0}(i)\right) & & \text { for } p=2
\end{aligned}
$$


Proof: The map of extensions

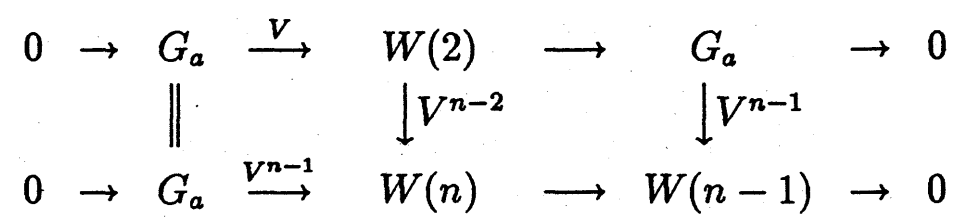

induces a map of spectral sequences

$$
\begin{array}{cccc}
E_{2}^{*, *}(n) \cong \mathrm{H}^{*}\left(W(n-1) ; \mathrm{H}^{*}\left(G_{a} ; k\right)\right) & \Longrightarrow & \mathrm{H}^{*}(W(n) ; k) \\
\left\lfloor V^{n-2 *}\right. & & \downarrow V^{n-2 *} \\
E_{2}^{*, *}(2) \cong & \mathrm{H}^{*}\left(G_{a} ; \mathrm{H}^{*}\left(G_{a} ; k\right)\right) & \Longrightarrow & \mathrm{H}^{*}(W(2) ; k) .
\end{array}
$$

By induction, we assume

$$
\mathrm{H}^{*}(W(n-1) ; k) \cong \bigotimes_{i=0}^{\infty} k\left[y_{n-2}(i+1)\right] \otimes E\left(x_{0}(i)\right)
$$

Since $V^{n-2 *} y_{j}(i+1)=y_{j-n+2}(i+1)$, and $V^{n-2 *} x_{j}(i)=x_{j-n+2}(i)$, where $y_{j}(i+1)=$ $x_{j}(i)=0$ for $j<0$, we get

$$
d_{2} x_{n-1}(i)=y_{n-2}(i+1) \text { modulo the ideal }\left(x_{0}(i)\right)
$$

from the naturality and from the result for $n=2$. Hence $E_{3}^{*, *}(n)$ is isomorphic to the formular in the theorem, and we see that $E_{3}^{*, *}(n) \cong E_{\infty}^{*, *}(n)$ by the same reason as in the case $n=2$.

The proof for the case $p=2$ is by similar arguments exchanging $y_{j}(i+1)$ with $x_{j}(i)^{2}$.

Corollary 3.3 The map $F^{*}$ on $\mathrm{H}^{*}(W(n) ; k)$ induced from the Frobenius map is injective.

Proof: This follows from the Theorem since $F^{*} x_{j}(i)=x_{j}(i+1)$ and $F^{*} y_{j}(i+1)=y_{j}(i+2)$.

\section{Higher Bockstein operations}

Recall that $\mathrm{H}^{*}(W(n) ; k)$ is generated by $y_{n-1}(i+1)$ and $x_{0}(i)$. We may and will hereafter assume that $y_{n-1}(i+1) \in \mathrm{H}^{2}(W(n) ; k)$ has a representative in $C^{2}(W(n+1), k)$ of the form

$$
Y=\partial^{1} x_{n}^{p^{i}}=\Delta x_{n}^{p^{i}}-\left(x_{n}^{p^{i}} \otimes 1+1 \otimes x_{n}^{p^{i}}\right)
$$


since $V^{n-1 *}\left(\triangle x_{n}\right)=\triangle x_{1}$ and $\partial^{2} \partial^{1}\left(x_{n}^{p^{i}}\right)=0$ in $C^{3}(W(n+1), k)$, so $Y$ is a cocycle. For $n=1$ we have the Bockstein $\beta x_{0}(i)=y_{0}(i+1)$. For $n \geq 1$ we define the higher Bockstein $\beta_{n}$ for $W(n)$ by: $\beta_{n} x_{0}(i)=y_{n-1}(i+1)$, setting $\beta=\beta_{1}$. In general

Definition 4.1 Let $G$ be an algebraic group defined over $k$. For an element $x \in \mathrm{H}^{1}(G ; k)$ and an integer $n \geq 1$ we define the higher Bockstein of $x$ to be an element $\beta_{n} x=y$ in $\mathrm{H}^{2}(G ; k)$ if there is a map $q: G \rightarrow W(n)$ of algebraic $k$-groups such that the induced map $q^{*}: \mathrm{H}^{*}(W(n) ; k) \rightarrow \mathrm{H}^{*}(G ; k)$ satisfies $q^{*} x_{0}(0)=x$ and $q^{*} y_{n-1}(1)=y$.

Theorem 4.2 Let $G$ be an algebraic $k$-group. For each element $x \in \mathrm{H}^{1}(G ; k)$ such that $\beta_{1}(x)=\cdots=\beta_{n}(x)=0$, then $\beta_{n+1}(x)$ is defined.

Proof: For an element $x \in \mathrm{H}^{1}(G ; k)$, let $\tilde{x} \in C^{1}(G, k)$ be a representative of $x$. Then $\partial^{1} \tilde{x}=0$ implies that $\tilde{x}$ is primitive and we get a Hopf algebra homomorphism:

$$
k\left[G_{a}\right] \cong k[\tilde{x}] \hookrightarrow k[G]
$$

which induces a homomorphism of algebraic groups $q: G \rightarrow G_{a}$ such that $q^{*} x(0)=x$. Hence the theorem is true for $n=1$.

Now suppose $\beta_{1} x=\cdots=\beta_{n} x=0$. The last equality implies there is an algebraic group homomorphism $q: G \rightarrow W(n)$ with $q^{*} x_{0}(0)=x$ and $q^{*} y_{n-1}(1)=0$ in $\mathrm{H}^{*}(G ; k)$. Let $\tilde{x} \in C^{1}(G ; k)$ be such that $\partial^{1} \tilde{x}$ represents $q^{*} y_{n-1}(1)$ in $C^{2}(G, k)$. Define a map

$$
\phi: k[W(n+1)] \rightarrow k[G]
$$

as follows: $\left.\phi\right|_{k\left[x_{0}, \ldots, x_{n-1}\right]}=q$ and $\phi\left(x_{n}\right)=\tilde{x}$. The map $\phi$ is a map of Hopf algebra such that $\phi^{*} x_{0}(0)=x$ and $\phi^{*} y_{n}(1):=\beta_{n+1} x$. This finishes the proof of the theorem.

As a consequence of this Theorem, we can explicitly write down $\beta_{n}$ in the cobar complex. For any sequence $I=\left(i_{0}, \ldots\right)$, with $i_{s} \geq 0$, for all $s \geq 0$, let $a^{I}$ denote $a_{0}^{i_{0}} a_{1}^{i_{1}} \ldots$. Take $\xi_{I J r} \in k$ such that

$$
(a \oplus b)_{r}=a_{r}+b_{r}+\sum \xi_{I J r} a^{I} b^{J}
$$

If $x \in \mathrm{H}^{1}(G ; k)$ and $\beta_{1} x=\cdots=\beta_{n} x=0$, then there are $x_{1}, \ldots, x_{n}$ such that $d x_{r}=$ $\sum \xi_{I J r} x^{I} \otimes x^{J}$ for $1 \leq r \leq n$ and we can define

$$
\beta_{n+1} x=\sum \xi_{I J n} x^{I} \otimes x^{J}
$$

QUESTION It is still an open question whether the higher Bockstein $\beta_{n}$ can be extended to all of $\mathrm{H}^{*}(G ; k)$.

We have the following nonvanishing lemma for the higher Bockstein. 
Lemma 4.3 Let $G$ be an algebraic $k$-group. Consider the spectral sequence induced from a central extension $0 \rightarrow G_{a} \rightarrow G \stackrel{\pi}{\rightarrow} G^{\prime} \rightarrow 1$. For any integer $n \geq 1$, if in the HochschildSerre's spectral sequence, $d_{2} x(0)=\beta_{n}\left(x^{\prime}\right) \neq 0$ for $x(0) \in \mathrm{H}^{1}\left(G_{a} ; k\right)$ and $x^{\prime} \in \mathrm{H}^{1}\left(G^{\prime} ; k\right)$. Then $\beta_{n+1}\left(\pi^{*} x^{\prime}\right) \neq 0$ in $\mathrm{H}^{*}(G ; k)$.

Proof: Since $\beta_{n}\left(\pi^{*} x^{\prime}\right)=0$ in $\mathrm{H}^{*}(G ; k)$, there exists a map $q_{n}: G \rightarrow W(n)$ inducing a map of extensions

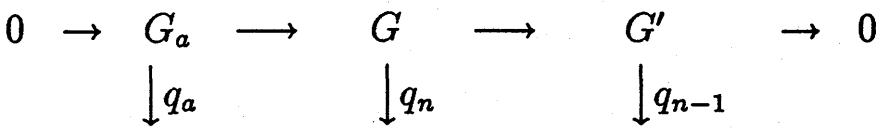

$$
\begin{aligned}
& 0 \rightarrow G_{a} \longrightarrow W(n) \longrightarrow W(n-1) \rightarrow 0
\end{aligned}
$$

with $q_{n-1}^{*} x_{0}(0)=x^{\prime}$. Since $q_{n}^{*} y_{n-2}(1)=\beta_{n-1}\left(x^{\prime}\right) \neq 0$ in the $E_{2}$ term of the spectral sequence associated to the first extension, we know that $q_{a}^{*} x_{n-1}(0) \neq 0$ in $\mathrm{H}^{*}\left(G_{a} ; k\right)$ since $d_{2} x_{n-1}(0)=y_{n-2}(1) \in \mathrm{H}^{2}(W(n-1), k)$. Hence $q_{a}^{*} y_{n-1}(1)=q_{a}^{*} \beta x_{n-1}(0) \neq 0$ in $E_{2}^{*, *}$. Since $y_{n-1}(1)$ is permanent, so is $q_{a}^{*} y_{n-1}(1)$ which is $\beta_{n}\left(\pi^{*} x^{\prime}\right)$.

\section{The group $V(n)$}

Every commutative affine algebraic group over $k$ whose underlying varity is an affine $n$ space is isogeneous to a product of Witt groups. I.e. it is an extension of a product of Witt groups by a finite abelian group. Those groups that are of interest to us in this work are the ones that are isomorphic as algebraic group to a product $\prod_{i}^{m} W\left(n_{i}\right)$, when $n_{i} \leq n_{i+1}$ and $\sum n_{i}=n$. For $n=m$ we get the additive vector group $G_{a}^{n}$ and for $m=1$ we get $W(n)$. See [6].

For each integer $n \geq 2$, let $V(n)$ be the commutative linear algebraic group isomorphic to a subgroup of the unipotent group $U(n)$ consisting of $n \times n$ upper triangular matrices such that each entry along an off diagonal is constant. More precisely, a matrix $\left[a_{i, j}\right] \in V(n)$ if $a_{i, j}=\delta_{i, j}$ for $i \geq j$, and $a_{i, j}=a_{i+r, j+r}$ for $i<j$ and $0 \leq r \leq n-i$. The coordinate algebra $k[V(n)]$ is a polynomial algebra $k\left[a_{1}, \ldots, a_{n-1}\right]$ with comultiplication $\Delta a_{i}=\sum_{j=0}^{i} a_{j} \otimes a_{i-j}$, where, by convension, $a_{0}=1 . V(n)$ is the so called big Witt group of length $n$, or Witt group at all prime simultaneously. It isomorphic as an algebraic group to a product of Witt groups.

$$
V(n) \cong \prod_{p \nmid i} W\left(r_{i}\right)
$$

where for each $i, r_{i}$ is the smallest positive integer such that $p^{r_{i}} \geq n / i$. See [6] chapter 5. This decomposition, together with the rational cohomology of $W(n)$ computed in the previous section immediately yield $\mathrm{H}^{*}(V(n) ; k)$. However we can compute $\mathrm{H}^{*}(V(n) ; k)$ directly. Using the higher Bockstein operation we will prove (9) by showing that there is a tensor decomposition of $\mathrm{H}^{*}(V(n) ; k)$ in terms of $\mathrm{H}^{*}(W(m) ; k)$. 
Like in $W(n)$, there exist the Frobenius, the restriction and the shift homomorphisms for $V(n)$ and we will also denote them by $F, R$ and $V$ respectively. These maps induce various extensions, in particular

$$
0 \rightarrow G_{a} \rightarrow V(n+1) \rightarrow V(n) \rightarrow 0 .
$$

with the associated Hochschild-Serre's spectral sequence

$$
E_{2}^{p, q}(n+1)=\mathrm{H}^{p}\left(V(n) ; \mathrm{H}^{q}\left(G_{a} ; k\right)\right) \Longrightarrow \mathrm{H}^{p+q}(V(n+1) ; k) .
$$

Let us denote by $S(n)$ (resp. $E(n)$ ) the symmetric algebra $S\left(\oplus k y_{n}(i+1)\right.$ ) (resp. exterior algebra $\left.E\left(\oplus k x_{n}(i)\right)\right)$. For $p=2$, let $y_{n}(i+1)=x_{n}(i)^{2}$.

Theorem 5.1 For all $n \geq 2$,

(a) $V(n) \cong \prod_{p \nmid i} W\left(r_{i}\right)$

(b) $\mathrm{H}^{*}(V(n) ; k) \cong \otimes_{p \nmid i=1}^{n-1} S\left(p^{r_{i}-1} i\right) \otimes E(i)$,

where $r_{i}$ is the smallest integer such that $p^{r_{i} i} \geq n$ and $\beta_{r_{i}} x_{i}(j)=y_{p^{r_{i}-1_{i}}}(j+1)$.

The proof of the Theorem follows from the following Lemmas which may be useful for other results. Let $\mathrm{G}$ be a unipotent algebraic group obtained from an extension of a product of Witt groups by $G_{a}$.

$$
0 \rightarrow G_{a} \rightarrow G \rightarrow \prod_{i=1}^{m} W\left(s_{i}\right) \rightarrow 0
$$

If we write $k\left[W\left(s_{i}\right)\right]=k\left[x_{i, 0}, \ldots, x_{i, s_{i}-1}\right]$ and $k\left[G_{a}\right]=k[x]$, then their cohomologies are $\mathrm{H}^{*}\left(W\left(s_{i}\right) ; k\right)=\otimes_{j=0}^{\infty} k\left[y_{i, s_{i}-1}(j+1)\right] \otimes E\left(x_{i, 0}(j)\right)$, and $\mathrm{H}^{*}\left(G_{a} ; k\right)=\otimes_{j=0}^{\infty} k[y(j+1)] \otimes E(x(j))$ respectively, by Theorem 3.2 .

Lemma 5.2 In the spectral sequence induced from the extension (12);

(1) If $d_{2} x(0)=0$, then $G \cong\left(\Pi W\left(s_{i}\right)\right) \times G_{a}$,

(2) If $d_{2} x(0)=y_{j, s_{j}-1}(1)$ for some $1 \leq j \leq m$, then $G \cong\left(\prod_{j \neq i} W\left(s_{i}\right)\right) \times W\left(s_{j}+1\right)$. 
Proof: Consider the coextension associated to the extension (12)

$$
k\left[x_{n}\right] \leftarrow k[G] \leftarrow \otimes k\left[W\left(s_{i}\right)\right] .
$$

If $d_{2} x(0)=0$, then $0 \neq x(0) \in \mathrm{H}^{1}(G ; k)$ induces a map

$$
\pi: G \rightarrow\left(\prod W\left(s_{i}\right)\right) \times G_{a}
$$

which induces an epimorphism in the coordinate algebras. Since $k[G]$ is polynomial, it also induces an isomorphism of groups by dimension counting argument.

Next consider the case $d_{2} x(0)=y_{j, s_{j}-1}(1)$. Since $y_{j, s_{j}-1}(1)=\beta_{s_{j}} x_{j, 0}(0)$, by Lemma 4.3 $\beta_{s_{j+1}} x_{j, 0}(0) \neq 0$ in $\mathrm{H}^{2}(G ; k)$. Let $\psi: G \rightarrow W\left(s_{j}+1\right)$ be the map defining $\beta_{s_{j}+1} x_{j, 0}(0)$. We get

$$
G \stackrel{\pi}{\longrightarrow}\left(\prod_{i \neq j} W\left(s_{i}\right)\right) \times W\left(s_{j}+1\right) .
$$

Since $d_{2} x(0)=y_{j, s_{j}-1}(1)=\beta_{s_{j}} x_{j, 0}(0)$. In the cobar complex $C^{2}(G)$ we have

$$
\partial^{1} x=\pi^{2}\left(\beta_{s_{j}} x_{j, 0}\right)=\pi^{2}\left(\partial^{1} x_{j, s_{j}}\right)=\partial^{1} \pi^{1} x_{j, s_{j}} .
$$

Therefore $\partial^{1}\left(x-\pi^{1} x_{j, s_{j}}\right)=0$ but $d_{2} x(0) \neq 0$. Hence $x=i^{1} \pi^{1} x_{j, s_{j}}$ in $k\left[G_{a}\right]$, for $i: G_{a} \rightarrow G$. This means $\pi^{*}$ is surjective and hence $\pi$ is an isomorphism of groups.

Lemma 5.3 Let $G$ be a commutative unipotent group defined in (12). Then in the associated spectral sequence

$$
d_{2} x(0)=\sum \mu_{i}(s) y_{i, s_{i}-1}(s), \quad \mu_{i}(s) \in k .
$$

Proof: Suppose $p \geq 3$. Write

$$
d_{2} x(0)=\sum \lambda_{i, j}(k, l) x_{i, 0}(k) x_{j, 0}(l)+\sum \mu_{i}(s) y_{i, s_{i}-1}(s),
$$

for $\lambda_{i, j}(k, l)$ and $\mu_{i}(s) \in k$. This means that there is an element $a \in C^{1}(G)$ such that $a$ belongs to the ideal $\left(x_{i, j}\right)$, i.e. the image of $a$ in $C^{1}\left(G_{a}\right)=0$ and

$$
\partial^{1}(x-a)=\sum \lambda_{i, j}(k, l) x_{i, 0}^{p^{k}} x_{j, 0}^{p^{l}}+\sum \mu_{i}(s)\left(\beta_{s_{i}-1} x_{i, 0}\right)^{p^{s}}
$$

in $C^{2}(G)$. Since $G$ is a commutative group, the coboundary $\partial^{1}$ must be cocommutative. This implies that $\partial^{1}(x-a)$ is invariant under the twist, $\tau(c \otimes d)=d \otimes c$, in $C^{2}(G)$. Therefore $\lambda_{i, j}(k, l)=\lambda_{j, i}(l, k)$. But $x_{i, 0}(k) x_{j, 0}(l)=-x_{j, 0}(l) x_{i, 0}(k)$ in $\mathrm{H}^{2}\left(\prod W\left(s_{i}\right) ; k\right)$, which forces $\lambda_{i, j}(k, l)=0$ for all $i, j, k, l$. Hence $d_{2} x(0)=\sum \mu_{i}(s) y_{i, s_{i}-1}(s)$. 
The case $p=2$ is proved by replacing $y_{i, 0}(k+1)$ by $x_{i, 0}(k) \otimes x_{i, 0}(k)$ and use similar argument as in the case $p>2$.

Proof of Theorem 5.1. Assume $p \geq 3$. It is clear that the lemma is true for $n=2$. Assume true for $n \geq 2$ and induct on $n$. The group $V(n+1)$ can be obtained from $V(n)$ by extension by $G_{a}$, i.e. it is the extension (12) with the following replacements: $G \leadsto V(n+1), s_{i} \leadsto r_{i}, p \nmid i, r_{i}$ the smallest positive integer such that $p^{r_{i}} i \geq n-1$, $x_{i, j} \leadsto x_{i p^{j}}$ and $x \leadsto x_{n}$. Recall that the weight $w\left(x_{i}(j)\right)=w\left(y_{i}(j)\right)=i p^{j}$, which, of course, is preserved by the differential. From Lemma 5.3 , we have

$$
d_{2} x_{n}(0)= \begin{cases}\mu y_{\frac{n}{p}}(1) & \text { if } p \mid n \\ 0 & \text { otherwise }\end{cases}
$$

because the other elements of the same degree are also of the same weight, hence they are all of the form $y_{\frac{n}{p^{s}}}(s)$ for some $s \geq 2$. But these elements do not appear in the assumption (b) for $n-1$.

We will now show that $\mu \neq 0$. First take $n=p$, we will show that $V(p+1) \not G_{a}^{p}=$ $G_{a} \times \cdots \times G_{a}$. For simplicity in the notation, we denote a matrix $\left[a_{i j}\right] \in V(n)$ by its first row entries: $\left[a_{i, j}\right]=\left(1, a_{1}, \ldots, a_{n-1}\right)$. For $n+1=p+1$ consider the matrix $A=\left[a_{i j}\right]=(1,1,0, \ldots, 0) \in V(p+1)$. Then $A^{p}=(1,0, \ldots, 0,1) \neq I$, with the non trivial entries in position 1 and $p+1$. Hence $V(p+1)$ is not a product of $G_{a}$. Now, if $\mu=0$, by induction and Lemma 4.3 implies that $V(p+1)$ is a product of $G_{a}$, which leads to a contradiction.

Let $n+1=m p+1$ and let $\iota: V(p+1) \hookrightarrow V(m p+1)$ be an inclusion of $V(p+1)$ into $V(m p+1)$ defined as

$$
\iota\left(a_{1}, \ldots, a_{p}\right)=(1, \underbrace{0, \ldots, 0, a_{1}}_{m}, \underbrace{0, \ldots, 0, a_{2}}_{m}, \ldots, \underbrace{0, \ldots, 0, a_{p}}_{m})
$$

By the naturality with respect to $\iota$ of the spectral sequences, $d_{2} x_{p}(0)=y_{1}(1)$ induces $d_{2} x_{m p}(0)=y_{m}(1)$. The Frobenius $F^{*}$ then implies

$$
d_{2} x_{n}(i)= \begin{cases}y_{\frac{n}{p}}(i+1) & \text { if } p \mid n \\ 0 & \text { otherwise. }\end{cases}
$$

This proves Theorem 5.1 (b) for the case $n+1$. The Bockstein is given by Lemma 4.3 . Part (a) follows from Lemma 4.3. The case $p=2$ is proved similarly by replacing $y_{j}(i+1)$ with $x_{j}(i)^{2}$.

Remark 5.4 The subalgebra $k\left[x_{0}, x_{1}^{p^{s}}\right] \subset k\left[x_{0}, x_{1}\right]=k[W(2)]$ is a Hopf subalgebra. Hence there is a group $W_{s}(2)$ isogenic to $W(2)$. For the extension.

$$
0 \rightarrow G_{a} \rightarrow W_{s}(2) \rightarrow G_{a} \rightarrow 0
$$


the differential of the induced spectral sequence is $d_{2} x_{1}(0)=y_{0}(s+1)$. And hence

$$
\mathrm{H}^{*}\left(W_{s}(2) ; k\right) \cong\left(\otimes_{i=1}^{s} S\left(y_{0}(i)\right) \otimes_{j=s+1}^{\infty} S\left(y_{1}(j)\right)\right) \otimes_{k=0}^{\infty} E\left(x_{0}(k)\right),
$$

with $y_{0}(i)=\beta x_{0}(i-1)$ and $y_{1}(i)=\beta_{2} x_{0}(j-1)$.

\section{Frobenius Kernel and the Steenrood Algebra}

Let $r$ be a positive integer and let $G_{r}$ be the $r^{\text {th }}$ Frobenius kernel of an algebraic $k$-group $G$, i.e. it is the kernel of the $r^{\text {th }}$ power of the Frobenius homomorphism

$$
0 \longrightarrow G_{r} \longrightarrow G \stackrel{F^{r}}{\longrightarrow} G \longrightarrow 0 .
$$

It is easy to obtain the similar results as in Sections 3 to 5 for the rational cohomology $\mathrm{H}^{*}\left(G_{r} ; k\right)$. For example

$$
\mathrm{H}^{*}\left(W(n)_{r} ; k\right) \cong \bigotimes_{i=0}^{r-1} k\left[y_{n-1}(i+1)\right] \otimes E\left(x_{0}(i)\right),
$$

and $\beta_{n}\left(x_{0}(i)=y_{n-1}(i+1)\right.$.

Let $G(n)$ be the subgroup of the unipotent group $U(n)$ such that a matrix $\left[a_{i, j}\right] \in G(n)$ if $a_{i, j}=\delta_{i, j}$ for $i \geq j$ and $a_{i, j}^{p^{r}}=a_{i+r, j+r}$ for $i<j$ and $0 \leq r \leq n-i$. The coordinate ring $k[G(n)]$ is a polynomial algebra $k\left[a_{1}, \ldots, a_{n-1}\right]$ with the comultiplication

$$
\triangle a_{i}=\sum_{j=0}^{i} a_{j} \otimes a_{i-j}^{p^{j}} .
$$

On the otherhand, let $P(n)$ be the finite dimensional subalgebra of the Steenrod algebra generated by the reduced powers $P^{p^{0}}, \ldots, P^{p^{n}}$. Its dual Hopf algebra is

$$
P(n)^{*} \cong k\left[\xi_{1}, \ldots, \xi_{n+1}\right] /\left(\xi_{1}^{p^{n+1}}, \xi_{2}^{p^{n}} \ldots, \xi_{n+1}^{p}\right)
$$

with $\triangle \xi_{i}=\sum_{j=0}^{i} \xi_{j} \otimes \xi_{i-j}^{p^{j}}$. There is a Hopf algebra epimorphism by (3.3) in [4].

$$
k\left[G(n)_{n-1}\right] \rightarrow P(n-2)^{*} .
$$

Therefore $\mathrm{H}^{*}\left(G(n)_{n-1} ; k\right)$ is important in homotopy theories. However the computations seem difficult except for $p=2$ and $n \leq 3$ which we now show. 
Consider the spectral sequence arises from the extension $1 \rightarrow G_{a 2} \rightarrow G(3)_{2} \rightarrow G_{a 2} \rightarrow 1$

$$
E_{2}^{* * *} \cong k\left[x_{1}(0), x_{2}(0), x_{1}(1), x_{2}(1)\right],
$$

with $d_{2} x_{2}(0)=x_{1}(0) x_{1}(1)$ and $d_{2} x_{2}(1)=x_{1}(1) x_{1}(2)=0$. Therefore we have

$$
E_{3}^{*, *} \cong k\left[x_{1}(0), x_{1}(1)\right] /\left(x_{1}(0) x_{1}(1)\right) \otimes k\left[x_{2}(0)^{2}, x_{2}(1)\right] .
$$

The next differential is (see A1, 5.2 in [5])

$$
\begin{aligned}
d_{3} x_{2}(0)^{2} & =d_{3} \widetilde{S q}^{1} x_{2}(0)=\widetilde{S q}^{1}\left(x_{1}(0) x_{1}(1)\right) \\
& =\widetilde{S q}^{1} x_{1}(0) \widetilde{S q}^{0} x_{1}(1)+\widetilde{S q}^{0} x_{1}(0) \widetilde{S q}^{1} x_{1}(1) \\
& =x_{1}(1)^{3} .
\end{aligned}
$$

Therefore we get

$$
E_{4}^{*, *} \cong k\left[x_{2}(0)^{4}, x_{2}(1)\right] \otimes\left(k\left[x_{1}(0), x_{1}(1)\right] /\left(x_{1}(0) x_{1}(1), x_{1}(1)^{3}\right) \oplus k\left[x_{1}(0)\right] x_{1}(0) x_{2}(0)^{2}\right),
$$

and this is isomorphic to $E_{\infty}^{* * *}$. This result is essentially obtained by Liuevicius. See for example, 3.1.24 in [5], where their notation is the following $h_{10}=x_{1}(0), h_{11}=x_{1}(1)$, $w=x_{2}(0)^{4}$ and $v=x_{1}(0) x_{2}(0)^{2}$, and

$$
\mathrm{H}^{*}\left(G(3)_{2} ; k\right) \cong \operatorname{Ext}_{P(1)^{*}}(k ; k) \otimes k\left[x_{2}(1)\right] .
$$

\section{References}

[1] E. Cline, B. Parshall, L. Scott, van de Kallen, Rational and generic cohomology, Invent. Math. 39 (1977), 143-163.

[2] N. Jacobson, Basic Algebra II.

[3] M. Kaneda, N. Shimada, M. Tezuka and N. Yagita, Cohomology of infinitesimal algebraic groups, Math. Z. 205 (1990), 61-95.

[4] M. Kaneda, N. Shimada, M. Tezuka and N. Yagita, Representations of the Steenrod algebra, to appear in J. of Algebra.

[5] D. Ravenel, Complex Cobordism and Stable Homotopy Groups of Spheres, (1986), Academic Press inc.

[6] J. P. Serre, Algebraic Groups and Class Fields. 
Nobuagi Yagita

Musashi Institute of Technology

Tamazutsumi, Setagaya-Ku

Tokyo 158, JAPAN
Chariya Peterson

Department of Mathematics Northwestern University

Evanston, IL 60208-2033

chariya@math.nwu.edu 